\title{
Inhomogeneous broadening in non-interacting nonlocal plasmonic ensembles
}

\author{
Tserkezis, Christos; Maack, Johan Rosenkrantz; Liu, Z.; Wubs, Martijn; Mortensen, N. Asger
}

Published in:

Proceedings of 2016 10th International Congress on Advanced Electromagnetic Materials in Microwaves and Optics

Link to article, DOI:

10.1109/MetaMaterials.2016.7746402

Publication date:

2016

Document Version

Peer reviewed version

Link back to DTU Orbit

Citation (APA):

Tserkezis, C., Maack, J. R., Liu, Z., Wubs, M., \& Mortensen, N. A. (2016). Inhomogeneous broadening in noninteracting nonlocal plasmonic ensembles. In Proceedings of 2016 10th International Congress on Advanced Electromagnetic Materials in Microwaves and Optics (pp. 367-9). IEEE.

https://doi.org/10.1109/MetaMaterials.2016.7746402

\section{General rights}

Copyright and moral rights for the publications made accessible in the public portal are retained by the authors and/or other copyright owners and it is a condition of accessing publications that users recognise and abide by the legal requirements associated with these rights.

- Users may download and print one copy of any publication from the public portal for the purpose of private study or research.

- You may not further distribute the material or use it for any profit-making activity or commercial gain

- You may freely distribute the URL identifying the publication in the public portal 


\title{
Inhomogeneous Broadening in Non-interacting Nonlocal Plasmonic Ensembles
}

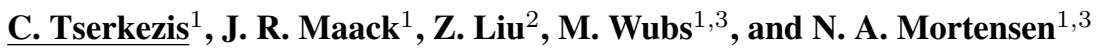 \\ ${ }^{1}$ Technical University of Denmark, Department of Photonics Engineering, Ørsteds Plads 343, 2800, Kgs. \\ Lyngby, Denmark \\ ${ }^{2}$ University of California, San Diego, Department of Electrical and Computer Engineering, 9500 Gilman \\ Drive, CA 92093, La Jolla, U.S.A. \\ ${ }^{3}$ Technical University of Denmark, Center for Nanostructured Graphene, 2800, Kgs. Lyngby, Denmark \\ ctse@fotonik.dtu.dk
}

\begin{abstract}
The importance of inhomogeneous broadening due to the size dependence of plasmon resonances in few-nm metallic particle ensembles is investigated through different models describing the nonlocal optical response of plasmonic nanospheres. Modal shifts and plasmon line broadening are shown to become important within the first-order correction to classical electrodynamics provided by the hydrodynamic Drude model, but turn out to be less prominent once additional single-particle size-dependent damping mechanisms are accounted for through the recently developed Generalized Nonlocal Optical Response theory. Our work is therefore expected to provide insight and facilitate the design of nanoscale spectroscopy experiments.
\end{abstract}

\section{INTRODUCTION}

In spite of the enormous success and popularity of plasmonics as a prominent field in modern nanotechnology [1], the realization of novel optical devices for visible light is still hindered by Ohmic losses [2] and Landau damping near the surface of the metallic components [3]. For relatively large plasmonic nanoparticles (NPs), this kind of homogeneous broadening is successfully captured by the common local response approximation (LRA) of classical electrodynamics, but size-dependent damping (SDD) corrections become necessary as NP size reduces [4]. More importantly, it is now well known that, for few-nm NPs, LRA also fails to predict the frequency position of plasmon resonances, because nonclassical effects such as nonlocality and electron spill-out become relevant $[5,6]$. It is therefore expected that in ensembles of such plasmonic NPs inhomogeneous broadening due to size distribution, which has been so far overlooked, will become important [7]. This effect is studied here theoretically, through simulations based on either the more common hydrodynamic Drude model (HDM) or the recently developed Generalized Nonlocal Optical Response (GNOR) [8] theory. While both models assume NP hard-wall boundaries, for which inclusion of nonlocality can only lead to resonance blueshifts as compared to LRA, our analysis is in fact independent of the nonclassical model employed, and holds even in situations where resonance redshifts prevail.

\section{INHOMOGENEOUS PLASMON BROADENING DUE TO NONLOCALITY}

The origin of inhomogeneous broadening, if observed in practice, is described in Fig. 1, where we plot with solid lines the frequency $(\omega)$ of the dominant dipolar plasmonic resonance sustained by a small metallic sphere of radius $R$ in air, described by a simple local, frequency-dependent Drude dielectric function $\varepsilon(\omega)=1-\omega_{\mathrm{p}}^{2} /\left(\omega^{2}+i \omega \gamma\right)$ as a function of its size. Here, $\omega_{\mathrm{p}}$ is the plasma frequency of the bulk metal, and $\gamma$ is the damping rate, taken equal to $0.01 \omega_{\mathrm{p}}$. It is immediately clear that, while for small NP sizes LRA (red line) predicts a saturation of the mode frequency at the quasistatic result $\omega_{\mathrm{p}} / \sqrt{3}$ [1], this ceases to be true once nonlocal effects are taken into account with any nonclassical theory, such as HDM (blue line in Fig. 1) [6]. Within HDM, nonlocality is introduced through a wavevector-dependent longitudinal component of the dielectric function, with an additional term $-\beta^{2} q^{2}$ added to the denominator of the Drude formula, where $q$ is the wavenumber and $\beta$ the hydrodynamic parameter, connected to the metal Fermi velocity $v_{\mathrm{F}}\left(=1.39 \cdot 10^{6} \mathrm{~m} \mathrm{~s}^{-1}\right.$ here $)$ through $\beta=\sqrt{3 / 5} v_{\mathrm{F}}$. To ensure that our analysis can be 
generalized to any metal, in what follows we normalize frequencies to $\omega_{\mathrm{p}}$, and radii to the corresponding plasmon wavelength $\lambda_{\mathrm{p}}=2 \pi c / \omega_{\mathrm{p}}$, where $c$ is the velocity of light in vacuum. Typical extinction spectra (with extinction values corresponding to the right-hand axis in Fig. 1) are shown by red (LRA) and blue (HDM) dotted lines for three NP sizes, $R / \lambda_{\mathrm{p}}=0.008,0.009$, and 0.01 . While in the local case the plasmon resonances appear at the same frequency, and with almost the same linewidth, HDM predicts not only size-dependent frequency shifts, but also differences in the linewidth and magnitude of the resonances. It is therefore expected that in a large collection of many (non-interacting) such NPs, the overlap of individual modes will lead to significant broadening of the resonance characterizing the entire ensemble.

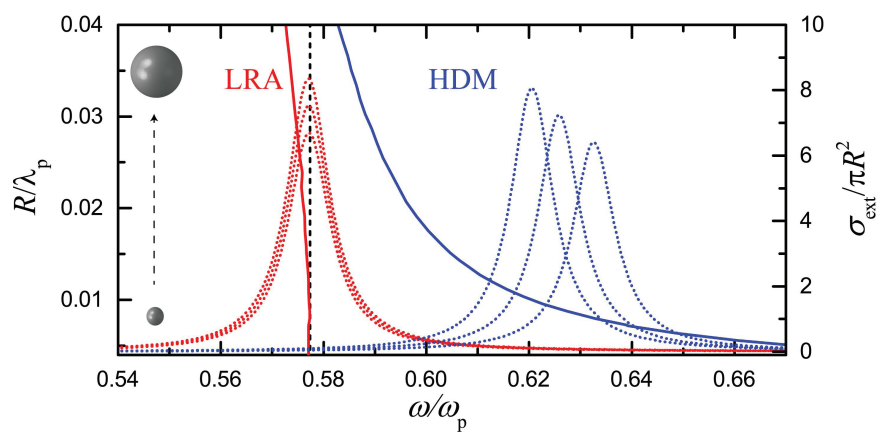

Fig. 1: Resonance frequency $\omega$ (normalized to the plasma frequency $\omega_{\mathrm{p}}$ ) of the dipolar plasmon mode of a metallic nanosphere in air, as a function of its radius $R$ (left vertical axis, normalized to the plasmon wavelenght $\lambda_{\mathrm{p}}$ ), as calculated with the LRA (red solid line) and HDM (blue solid line) models. Normalized extinction spectra (right vertical axis) for three NP sizes $R / \lambda_{\mathrm{p}}=0.008,0.009$, and 0.01 are shown by red and blue dotted lines (increasing in magnitude as NP size increases). The black dashed line denotes the prediction of the quasistatic approximation.

The effect of ensemble averaging is explored in Fig. 2, where we consider a collection of $N=1000$ NPs similar to the ones described above, following the normal size distribution function of the inset. The averaged spectra (solid lines) are compared to the corresponding spectra for a single NP of the mean size in the ensemble (open dots), which corresponds to the $\delta$-function size distribution shown by the dots in the inset. Clearly, while LRA predicts that the averaged spectrum is described extremely well, both in resonance frequency and linewidth, by just considering the single mean-size NP, the situation is much different within HDM. As expected from Fig. 1, both resonance frequency and linewidth become significantly different from those of the mean-size NP once nonlocal corrections are introduced, meaning that fabrication and measurements on such small plasmonic NPs must be treated with care. HDM however is not a complete model, as it fails to predict the additional damping observed in experiments for decreasing NP size. This is usually included phenomenologically with a SDD model [4], in which the damping parameter $\gamma$ of the Drude dielectric function becomes size dependent: $\gamma \rightarrow \gamma+A / R$, where $A$ is a constant. The predictions of SDD in our case are shown in Fig. 2 by black lines, and represent in principle a significant homogeneous broadening, but no averaging-related effect, as expected for any local model. The recent GNOR theory [8], on the other hand, explained for the first time the known SDD as an effect of nonlocal response. It accounts for both nonlocal frequency shifts and damping due to small NP size, and can be therefore considered as a more general model for the optical response of few-nm NPs. The resulting averaged spectra (green lines in Fig. 2) show that inhomogeneous broadening becomes less important once single-particle damping is properly considered. This conclusion provides additional flexibility to state-of-the-art experiments, since it indicates that few-nm NPs can be fabricated and studied in large ensembles without observing any strong effects due to inhomogeneous broadening. At the same time, this facilitates the design of far-field experiments for the observation and understanding of nonlocal effects, as a more easily accessible alternative to the common focusedelectron-beam studies [9]. Extensive simulations for more realistic size distributions of non- or weakly-interacting noble-metal NPs show that in these situations inhomogeneous broadening tends to be almost totally concealed by single NP damping, as long as the distribution function is not too wide [7]. A simple analytical formula, which relates plasmon linewidths to the statistical properties of the distribution function, can be immediately applied in every occasion to conclude whether inhomogeneous broadening will be observable or not [7]. 


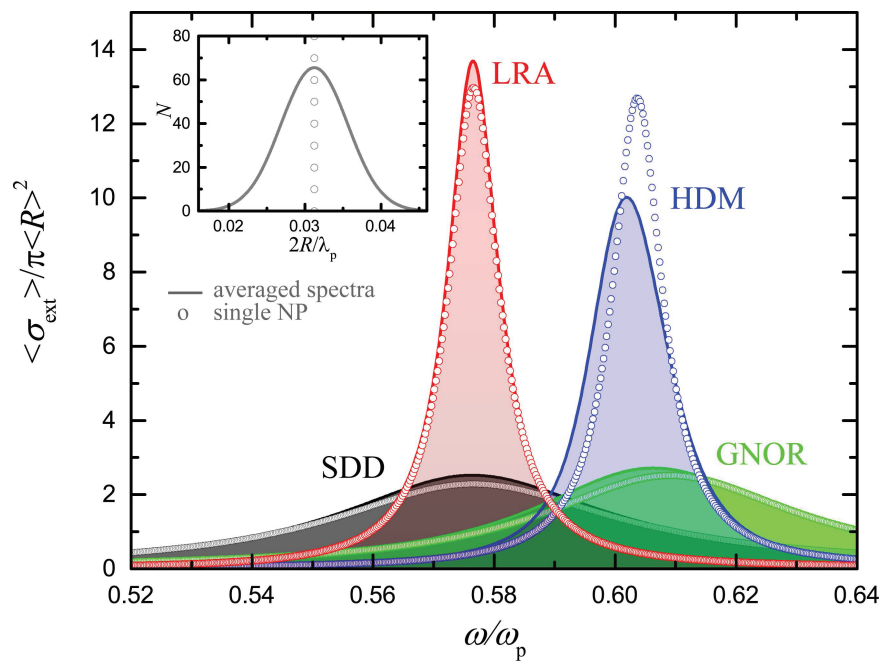

Fig. 2: Averaged normalized extinction spectra (solid lines) for $N=1000$ plasmonic NPs following the size distribution function shown in the inset, calculated within the LRA (red line), HDM (blue line), SDD (black line) and GNOR (green line) models. The average NP diameter is $2\langle R\rangle / \lambda_{\mathrm{p}}=0.031$. Open dots correspond to the spectra of a single NP of this mean size.

\section{CONCLUSION}

The importance of inhomogeneous broadening in ensembles of few-nm non-interacting plasmonic NPs was studied theoretically, starting from the common LRA approximation and increasing complexity to include both SDD effects and nonlocality-driven frequency shifts nonlocality. A description within the GNOR model, which takes both effects into account, predicts that inhomogeneous broadening shall not be large in most situations of practical interest, thus allowing to interpret far-field nanoscale measurements on the basis of the ensemble meansize NP, and design novel experiments for the investigation of the optical response of small plasmonic structures.

\section{ACKNOWLEDGEMENTS}

C. T. was supported by funding from the People Programme (Marie Curie Actions) of the European Union's Seventh Framework Programme (FP7/2007-2013) under REA grant agreement number 609405 (COFUNDPostdocDTU). We gratefully acknowledge support from the Villum Foundation via the VKR Centre of Excellence NATEC-II and from the Danish Council for Independent Research (FNU 1323-00087). The Center for Nanostructured Graphene is sponsored by the Danish National Research Foundation, Project DNRF103.

\section{REFERENCES}

[1] S.A. Maier, Plasmonics: Fundamentals and Applications, New York, U.S.A.: Springer, 2007.

[2] J.B. Khurgin, "How to deal with the loss in plasmonics and metamaterials," Nature Nanotechnology, vol. 10, p. $2,2015$.

[3] L.D. Landau and E.M. Lifshits, Electrodynamics of Continuous Media, 2nd edition, Oxford, England: Pergamon Press, 1984.

[4] U. Kreibig and L. Genzel, "Optical absorption of small metallic particles," Surface Science, vol. 156, p. 678, 1985.

[5] M.S. Tame, K.R. McEnery, S.K. Özdemir, J. Lee, S.A. Maier, and M.S. Kim, "Quantum plasmonics," Nature Physics, vol. 9 , p. 329, 2013.

[6] S. Raza, S.I. Bozhevolnyi, M. Wubs, and N.A. Mortensen, "Nonlocal optical response in metallic nanostructures," Journal of Physics: Condensed Matter, vol. 27, p. 183204, 2015.

[7] C. Tserkezis, J.R. Maack, Z. Liu, M. Wubs, and N.A. Mortensen, "Robustness of the far-field response of nonlical plasmonic ensembles," Scientific Reports, vol. 6, p. 28441, 2016.

[8] N.A. Mortensen, S. Raza, W. Wubs, T. Sondergaard, and S.I. Bozhevolnyi, "A generalized non-local optical response theory for plasmonic nanostructures," Nature Communications, vol. 5, p. 3809, 2014.

[9] S. Raza, S. Kadkhodazadeh, T. Christensen, M. Di Vece, W. Wubs, N.A. Mortensen, and N. Stenger, "Multipole plasmons and their disappearance in few-nanometre silver nanoparticles," Nature Communications, vol. 6, p. 8788, 2015. 